\title{
Consumo de alcohol y lesiones: estudio en población atendida en servicios de urgencias de la ciudad de Xalapa, Veracruz, México
}

\author{
María del Carmen Gogeascoechea-Trejo,' Silvia María Méndez-Maín, ${ }^{2}$ Guillermina Natera-Rey, ${ }^{3}$ \\ María Sobeida Leticia Blázquez-Morales,' Xóchitl de San Jorge-Cárdenas,' Sandra Treviño-Siller ${ }^{4}$
}

Artículo original

\section{ABSTRACT}

\section{Introduction}

Excessive alcohol consumption has escalated in recent decades, primarily in adolescents over 15 years of age. This situation can lead to an increase in social and health problems such as intentional violence-related injuries or unintentional injuries caused by accidents.

\section{Objective}

To identify the association between alcohol and injuries in patients treated in the emergency department and its relationship with certain sociodemographic characteristics.

\section{Method}

Cross-sectional study conducted in the emergency department of two hospitals in Xalapa, Veracruz (Mexico), between September and December 2013. Injured patients aged 15 years and over were administered a questionnaire. Logistic regression analyses were performed in order to calculate the odds ratio.

\section{Results}

Among the 505 patients surveyed, $28.9 \%$ reported having consumed alcohol prior to injury. Patients who used alcohol $(O R=2.42,95 \% \mathrm{Cl}$ : 1.36-4.31) and those who were men $(\mathrm{OR}=2.33,95 \% \mathrm{Cl}: 1.12-4.84)$ had a higher probability of severe injuries. Patients injured during night hours (OR $=3.56,95 \% \mathrm{Cl}: 2.20-5.77$ ) aged 15 to 29 years old $(\mathrm{OR}=1.68,95 \% \mathrm{Cl}: 1.05-2.70)$, and in the range between 6 to 15 cups of alcohol consumed (OR $=4.40,95 \% \mathrm{Cl}: 2.17-8.90)$ showed a higher probability of violence-related injuries.

\section{Discussion and conclusion}

The study showed the relationship between alcohol consumption and injuries. It is necessary to develop public policies that promote responsible drinking. These policies should emphasize controlling the harmful use of alcohol, particularly during weekends and at night, in order to contribute to the prevention of injuries and its complications, which can sometimes be fatal.

Key words: Alcohol, injuries, violence, accidents, emergency department.

\section{RESUMEN}

\section{Introducción}

El abuso de bebidas alcohólicas ha aumentado en las últimas décadas, principalmente en la población mayor de 15 años, lo que puede provocar un incremento de los problemas sociales y de salud, entre los que destacan las lesiones intencionales por violencia o no intencionales por accidentes.

\section{Objetivo}

Identificar la asociación entre consumo de alcohol, lesiones y características sociodemográficas en pacientes atendidos en el servicio de urgencias.

\section{Método}

Estudio transversal, en el servicio de urgencias de dos hospitales de Xalapa, Veracruz (México), entre septiembre y diciembre de 2013. Se aplicó un cuestionario a pacientes lesionados mayores de 15 años. Como medida de asociación se realizaron análisis de regresión logística para estimar las probabilidades mediante Odds Ratio (OR).

\section{Resultados}

Participaron 505 pacientes, $28.9 \%$ reportó haber consumido alcohol antes de la lesión. Quienes consumieron alcohol $(\mathrm{OR}=2.42$, IC95\% 1.36-4.31) y los hombres (OR $=2.33$, IC95\% 1.12-4.84) presentaron mayor probabilidad de sufrir lesiones graves. Pacientes lesionados en horario nocturno (OR $=3.56$, IC95\% 2.20-5.77), con edad entre 15 a 29 años $(O R=1.68, I C 95 \% 1.05-2.70)$ y el consumo de seis a 15 copas de alcohol $(O R=4.40$, IC95\% 2.17-8.90), mostraron mayor probabilidad de sufrir lesiones por violencia.

\section{Discusión y conclusión}

Se demostró la asociación del consumo de alcohol con la producción de lesiones. Es necesario que las políticas públicas estén destinadas a fomentar un consumo responsable de alcohol y enfatizar en el control de su uso nocivo que se realiza particularmente en fines de semana y en horario nocturno, para coadyuvar a la prevención de lesiones y sus complicaciones, que en ocasiones pueden ser fatales.

Palabras clave: Alcohol, lesiones, violencia, accidentes, servicio de urgencias.

Instituto de Ciencias de la Salud. Universidad Veracruzana. Xalapa, Ver., México.

Instituto Investigaciones Histórico Sociales. Universidad Veracruzana. Xalapa, Ver., México.

Instituto Nacional de Psiquiatría Ramón de la Fuente Muñiz. Ciudad de México.

Instituto Nacional de Salud Pública. Cuernavaca, Morelos. México.

Correspondencia: María del Carmen Gogeascoechea-Trejo. Instituto de Ciencias de la Salud, Universidad Veracruzana. Av. Luis Castelazo Ayala s/n, Xalapa, Ver., México. Tel: (228) 841 - 8925. E-mail: cgogeascoechea@uv.mx, mcgoge@hotmail.com

Recibido primera versión: 13 de mayo de 2015. Segunda versión: 15 de octubre de 2015. Tercera versión: 5 de febrero de 2016 . Aceptado: 16 de febrero de 2016. 


\section{INTRODUCCIÓN}

El abuso de bebidas alcohólicas ha aumentado en las últimas décadas, principalmente entre la población adolescente mayor de 15 años, lo que puede provocar un incremento de los problemas sociales y de salud, entre los que destacan las lesiones. ${ }^{1,2}$ El consumo de alcohol ha sido identificado como un factor que contribuye de manera significativa a la carga global de la enfermedad, así como para las lesiones no intencionales causadas por accidentes y las lesiones intencionales, ocasionadas por la violencia provocada por terceros o autoinfligida. $^{3}$

El uso nocivo del alcohol afecta a las personas ya que altera la capacidad de atención, la toma de decisiones para responder adecuada y oportunamente ante los estímulos del medio y disminuye la percepción de riesgo, lo que favorece una mayor incidencia de comportamientos riesgosos que pueden culminar en lesiones, con el consecuente impacto en los costos directos en los servicios de salud debido a que algunas de estas lesiones ameritan hospitalización, uso de quirófanos y otros servicios para su atención, sin contar con las complicaciones que, muchas veces, tienen consecuencias fatales para el individuo. ${ }^{1}$

El Informe Mundial de la Situación sobre Alcohol y Salud 2014, señala que 3.3 millones de personas murieron en el mundo durante 2012 debido al uso nocivo del alcohol y que éste es un factor causal de más de 200 enfermedades y de lesiones. Asimismo, menciona que 5.1\% de la carga mundial de enfermedades y lesiones -medida en años de vida ajustados por discapacidad (AVAD)- es atribuible al consumo de alcohol y de éste, $20.4 \%$ corresponde a lesiones no intencionales y $10.3 \%$ a lesiones intencionales. La región de las Américas ocupa el segundo lugar después de Europa, tanto en el consumo de alcohol per capita como en la tasa de consumo episódico de alcohol, definido como la ingesta de cinco copas o más por ocasión en los hombres y cuatro copas o más en las mujeres. ${ }^{1}$

El consumo episódico de alcohol provoca problemas agudos de salud en la población, particularmente en los jóvenes, entre los que destacan las lesiones, las cuales representan uno de los principales factores prevenibles asociados a la pérdida de años de vida activa y productiva., ${ }^{1,-6}$

En México, las diferentes encuestas nacionales sobre el tema reportan que la tendencia del consumo de alcohol va en aumento; $;, 7$ la Encuesta Nacional de Adicciones (ENA) muestra un incremento en el consumo de alcohol alguna vez en la vida, en el último año y en el último mes, tanto en hombres como en mujeres; sin embargo, en el consumo alto o episódico de alcohol, en los hombres entre 12 y 65 años de edad aumentó de $45 \%$ en 2008 a 47.2\% en 2011, mientras que esta forma de consumo en las mujeres prácticamente se mantuvo igual: $19.9 \%$ y $19.3 \%$ respectivamente. ${ }^{2}$

La vulnerabilidad a los efectos nocivos del alcohol difiere entre los grupos sociales definidos por género, edad, nivel educativo y socioeconómico, entre otros aspectos. ${ }^{8}$ En todas las sociedades donde se ha medido el consumo de alcohol, se ha mostrado que los hombres y las personas jóvenes de ambos sexos tienden a consumir alcohol con más frecuencia y en mayores cantidades, al mismo tiempo que presentan mayores comportamientos de riesgo, que podrían derivar en lesiones. ${ }^{9,10}$ Aunque ha sido objeto de controversia algunos autores señalan que las personas con mayor nivel educativo y socioeconómico tienden a consumir alcohol con mayor frecuencia y en pequeñas cantidades, a diferencia de los niveles más bajos cuyas ocasiones de consumo son menores pero en cantidades más altas. ${ }^{8,9}$

Diversos estudios auspiciados por la Organización Mundial de la Salud (OMS) ${ }^{11-19}$ en varios países, entre los que se encuentra México, han cuantificado el consumo de alcohol en las personas con lesiones, mostrando que la edad, el sexo y los hábitos de consumo son algunos factores que se asociaron con la probabilidad de presentar una lesión derivada de la violencia. Sin embargo, los mismos estudios mostraron diferencias entre los países participantes, principalmente en la proporción de pacientes que consumen alcohol y en la atención de personas lesionadas en los servicios de salud.

En lo que corresponde al Estado de Veracruz, la ENA 2008 registra un consumo de alcohol alto, de $21.8 \%$ en la población de 12 a 65 años, lo que resulta inferior a la media nacional de 26.6\%; sin embargo, la Encuesta de Salud y Nutrición (ENSANUT) 2012 reporta que 21.9\% de los adultos de 20 años o más refirió haber consumido alcohol en exceso por lo menos una vez en el último mes, ubicando a este Estado en la posición número 11 y por arriba de la estimación nacional que para este año fue de $20.9 \% .^{20}$

Debido a la importancia del problema del alcohol y las lesiones, se realizó este estudio en la ciudad de Xalapa, capital del Estado de Veracruz, México. Se trata del segundo municipio más poblado (457 928 habitantes) de los 212 que lo conforman, que se caracteriza por una actividad económica terciaria o de servicios relacionada con la administración pública y con las distintas universidades e instituciones de salud, lo que además explica una numerosa población flotante que se traslada a la capital para satisfacer sus necesidades en esos ámbitos. Al igual que en otras ciudades con estas características sociales, en Xalapa han proliferado numerosos establecimientos donde se ingieren bebidas alcohólicas, una gran cantidad de ellos dirigidos a la población joven. Este entorno, entre otros factores, explicaría el desarrollo de comportamientos de riesgo relacionados con el consumo de alcohol.

En este contexto, el propósito de este estudio fue identificar la asociación entre el consumo de alcohol, las lesiones y las características sociodemográficas en los pacientes que solicitaron atención en los servicios de urgencias de dos hospitales de Xalapa. 


\section{MÉTODO}

En la presente investigación se siguió el método utilizado en el Estudio Colaborativo de la Organización Mundial de la Salud (OMS) sobre Alcohol y Lesiones. ${ }^{21,22}$

Se realizó un estudio cuantitativo, transversal y correlacional, en el servicio de urgencias de dos hospitales públicos de los cinco que reciben urgencias en Xalapa, pertenecientes a los Servicios de Salud del Estado de Veracruz, que concentran el mayor porcentaje de pacientes con lesiones.

\section{Participantes}

Se eligieron a todos los pacientes de 15 años y más que solicitaron atención en el servicio de urgencias del hospital, por lesiones intencionales y no intencionales, durante las primeras seis horas después del evento que provocó la lesión. Se excluyeron a los pacientes que llegaron después de este período, a los que se encontraban muy intoxicados, a quienes se negaron a participar y a los que se retiraron del servicio antes de que pudieran ser entrevistados.

\section{Instrumento de medición}

Se empleó el Cuestionario sobre alcohol y lesiones aplicado y validado en México para el estudio colaborativo mencionado anteriormente, el cual consta de 60 ítems que incluye preguntas sobre las características sociodemográficas, el tipo y la causa de la lesión, el consumo de alcohol durante las seis horas previas a la lesión y la cantidad y tipo de bebidas consumidas durante este periodo.

\section{Procedimiento}

Previo al inicio de la recopilación de la información, los entrevistadores, estudiantes de medicina y enfermería en servicio social, fueron capacitados acerca de los procedimientos mediante un curso y un manual elaborados ex profeso por la investigadora responsable.

Los entrevistadores se ubicaron en el área de admisión del servicio de urgencias para identificar a los pacientes lesionados e invitarlos a participar en el estudio; les explicaron los objetivos y les entregaron el consentimiento informado garantizándoles la confidencialidad y el anonimato. Posteriormente procedieron a aplicar el cuestionario en un consultorio del servicio de urgencias o en el área de observación, mediante una entrevista cara a cara con una duración aproximada de 25 minutos. Los pacientes que no se encontraban en condiciones de contestar el cuestionario debido al mal estado físico ocasionado por la lesión y que requirieron hospitalización, fueron entrevistados posteriormente una vez que su estado se estabilizó.

La recopilación de información se llevó a cabo durante siete semanas en cada uno de los hospitales, diariamente y en los tres turnos (matutino, vespertino y nocturno), entre septiembre y diciembre de 2013.

\section{Variables}

Las variables consideradas en este estudio fueron: sexo, edad, escolaridad, día en que ocurrió la lesión, horario, consumo previo de alcohol, número de copas, intencionalidad de la lesión y gravedad de la misma.

Para los fines de este estudio se recodificaron las variables con el propósito de comparar los resultados con los reportados en otras investigaciones realizadas en los servicios de urgencias, 9,13-16,18,19,21 de manera que en la variable edad se establecieron dos grupos: de 15 a 29 años e igual o mayor a 30 años; asimismo, en la variable escolaridad se integraron dos categorías: las personas con estudios de bachillerato y menos y las que contaban con algún estudio de licenciatura y más. La variable intencionalidad de la lesión se categorizó en: lesiones intencionales (relacionadas con la violencia) y lesiones no intencionales (relacionadas con los accidentes). Respecto a la variable gravedad de la lesión, se consideraron como lesiones leves aquellas en las que no fue necesaria la hospitalización del paciente, y como lesiones graves las que requirieron hospitalización.

Para medir la variable consumo previo de alcohol se utilizó el autorreporte del consumo de alcohol por parte del lesionado, mediante la pregunta: "En las seis horas antes de que ocurriera su lesión, ¿ingirió alguna bebida alcohólica, aunque fuera sólo una copa?"

El análisis del número de copas en los pacientes que reportaron su consumo durante el período de seis horas previas a la lesión, se realizó mediante la suma del total de alcohol absoluto en mililitros, de acuerdo con el tamaño de las bebidas reportadas, considerando un tamaño estándar de $16 \mathrm{ml}$ como una copa.

\section{Análisis estadístico}

Se comparó la distribución de las variables de acuerdo con el consumo de alcohol previo a la lesión por medio de un análisis bivariado y se determinó la asociación entre las mismas utilizando la prueba estadística $\chi^{2}$.

Posteriormente, como medida de asociación se realizaron tres análisis de regresión logística con el método "Step Wise" para estimar las probabilidades mediante Odds Ratio (OR) con intervalos de confianza (IC) de 95\%, bajo el nivel de significancia de 0.05 para comprobar las tres hipótesis planteadas:

- El sexo, la edad, la escolaridad, y el día y hora en que ocurrió la lesión se asocian con el consumo de alcohol previo a la lesión.

- El sexo, la edad, la escolaridad, el consumo de alcohol previo a la lesión, y el día y hora en que ocurrió la lesión se asocian con la gravedad de la lesión. 


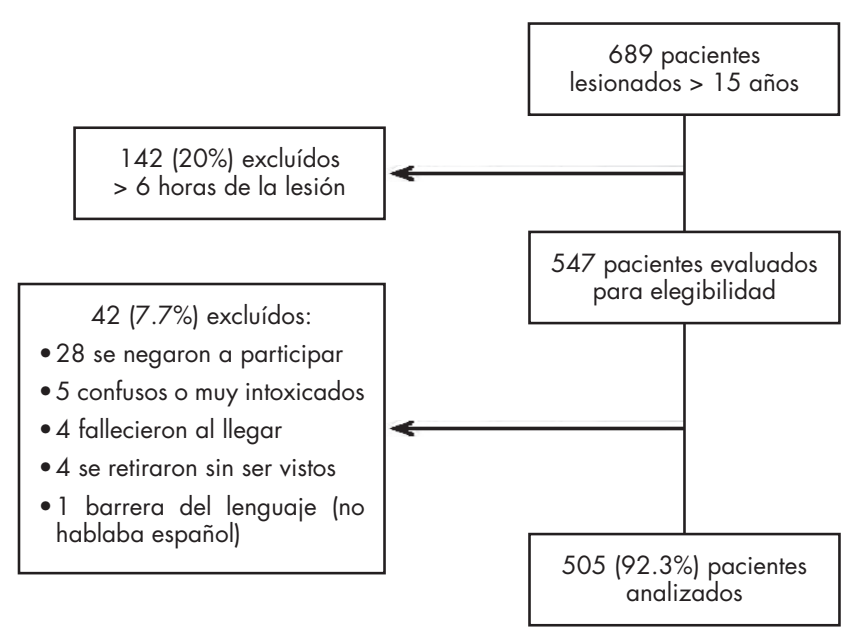

Figura 1. Diagrama de flujo de la participación de los pacientes.

- El sexo, la edad, la escolaridad, el número de copas de alcohol consumidas, y el día y hora en que ocurrió la lesión se asocian con la intencionalidad de la lesión.

En el primer análisis se consideró como variable criterio el consumo de alcohol previo a la lesión y como variables predictoras el sexo, la edad, la escolaridad, el día en que ocurrió la lesión y el horario. Para el segundo análisis, la variable criterio fue la gravedad de la lesión y como variables predictoras las mismas consideradas en el anterior análisis más la variable consumo previo de alcohol. Por último, en el tercer análisis, la variable criterio fue la intencionalidad de la lesión y como variables predictoras, además de las anteriores, se incluyó la variable número de copas con el fin de estimar las probabilidades, de acuerdo con la cantidad de alcohol consumida.

En todos los análisis el ajuste global se verificó con la prueba de Hosmer y Lemeshow, el cual se consideró adecuado cuando $p>0.05$. También se utilizó el estadístico de Wald, con un valor de $\mathrm{p}<0.05$, para determinar la relación entre las variables. El procesamiento se llevó a cabo en el paquete estadístico SPSS, versión 19 para Windows.

\section{RESULTADOS}

A los servicios de urgencias de los hospitales seleccionados llegaron 689 lesionados mayores de 15 años, pero sólo 547 cumplieron con los criterios de inclusión por lo que fueron invitados a participar en el estudio. De ellos, a 42 (7.7\%) no se les pudo realizar la entrevista por los siguientes motivos: 28 se negaron, cinco se encontraban confusos o muy intoxicados para cooperar, cuatro pacientes fallecieron, cuatro se retiraron del servicio de urgencias sin ser vistos y uno no pudo contestar las preguntas porque no hablaba español debido a que era indígena monolingüe. En resumen, participaron 505 pacientes, lo que significa una tasa de respuesta de $92.3 \%$, la mayoría fueron hombres $(63.8 \%)$, con una edad promedio de $34.9 \pm 17.7$ años (figura 1 ).

\section{Descripción de la población}

Del total de lesionados, 146 (28.9\%) reportaron consumo de alcohol en las seis horas previas a la ocurrencia de la lesión y 359 (71.1\%) dijeron no haber consumido. En el cuadro 1 se presenta la distribución de frecuencias de las variables consideradas en el estudio, de acuerdo con el consumo y no consumo de alcohol. Se observaron diferencias en cuanto al sexo ya que la mayor concentración de pacientes que consumieron alcohol fueron hombres.

A diferencia de los lesionados que no utilizaron alcohol, más de la mitad de los casos que lo habían consumido se ubicó en el grupo de edad de 15 a 29 años; asimismo cerca de una cuarta parte contaba con estudios de licenciatura. A pesar de que estas diferencias no resultan estadísticamente significativas en el análisis bivariado, tanto la variable de edad como la de escolaridad fueron incluidas en los análisis de regresión logística debido a que se consideraron importantes para entender la problemática (cuadro 1).

Con respecto al momento del evento, el fin de semana y el horario nocturno concentraron los mayores porcentajes de lesiones con consumo de alcohol. El porcentaje de lesiones por violencia fue alto en los pacientes que reportaron

Cuadro 1. Distribución de las variables de acuerdo con el consumo de alcohol previo a la lesión

\begin{tabular}{|c|c|c|c|c|c|}
\hline & \multicolumn{4}{|c|}{$\begin{array}{l}\text { Consumo de alcohol } \\
\text { previo a la lesión }\end{array}$} & \multirow[b]{3}{*}{$p$} \\
\hline & \multicolumn{2}{|c|}{ No } & \multicolumn{2}{|c|}{ Sí } & \\
\hline & $\%$ & $n=359$ & $\%$ & $n=146$ & \\
\hline \multicolumn{6}{|l|}{ Sexo } \\
\hline Mujer & 44.3 & 159 & 16.4 & 24 & 0.001 \\
\hline Hombre & 55.7 & 200 & 83.6 & 122 & \\
\hline \multicolumn{6}{|l|}{ Edad } \\
\hline 15 a 29 años & 48.7 & 175 & 58.2 & 85 & 0.053 \\
\hline$\geq 30$ años & 51.3 & 184 & 41.8 & 61 & \\
\hline \multicolumn{6}{|l|}{ Escolaridad } \\
\hline Bachillerato y menos & 83.8 & 301 & 76.7 & 112 & 0.060 \\
\hline Licenciatura y más & 16.2 & 58 & 23.3 & 34 & \\
\hline \multicolumn{6}{|l|}{ Día } \\
\hline Lunes a jueves & 56.5 & 203 & 36.3 & 53 & 0.001 \\
\hline Fin de semana & 43.5 & 156 & 63.7 & 93 & \\
\hline \multicolumn{6}{|l|}{ Horario } \\
\hline Diurno & 70.5 & 253 & 41.8 & 61 & 0.001 \\
\hline Nocturno & 29.5 & 106 & 58.2 & 85 & \\
\hline \multicolumn{6}{|c|}{ Intencionalidad de la lesión } \\
\hline Accidente & 85.8 & 308 & 55.5 & 81 & 0.001 \\
\hline Violencia & 14.2 & 51 & 44.5 & 65 & \\
\hline \multicolumn{6}{|l|}{ Gravedad de la lesión } \\
\hline Leve & 92.2 & 331 & 80.1 & 117 & 0.001 \\
\hline Grave & 7.8 & 28 & 19.9 & 29 & \\
\hline Total $(N=505)$ & 100.0 & 359 & 100.0 & 146 & \\
\hline
\end{tabular}


Cuadro 2. Variables predictoras del consumo de alcohol previo a la lesión, según análisis por regresión logística

\begin{tabular}{|c|c|c|c|c|c|}
\hline Variables & $\chi^{2}$ Wald & $\mathrm{gl}$ & Sig & OR & IC95\% \\
\hline \multicolumn{6}{|l|}{ Sexo } \\
\hline Hombre & 30.34 & 1 & 0.001 & 4.19 & $2.52-6.99$ \\
\hline Mujer & & & & 1 & \\
\hline \multicolumn{6}{|l|}{ Escolaridad } \\
\hline Licenciatura y más & 6.78 & 1 & 0.009 & 2.04 & $1.19-3.48$ \\
\hline Bachillerato y menos & & & & 1 & \\
\hline \multicolumn{6}{|l|}{ Día } \\
\hline Fin de semana & 13.54 & 1 & 0.001 & 2.23 & $1.45-3.42$ \\
\hline Lunes a jueves & & & & 1 & \\
\hline \multicolumn{6}{|l|}{ Horario } \\
\hline Nocturno & 28.39 & 1 & 0.001 & 3.17 & $2.08-4.88$ \\
\hline Diurno & & & & 1 & \\
\hline
\end{tabular}

Análisis de regresión logística: Razón de verosimilitud $=517.31 \chi^{2}(4)=90.05$ $p=0.001$. Hosmer y Lemeshow $\chi^{2}(7)=13.11 p=0.069$. Porcentaje global de varianza explicada $=78.0 \%$. Área bajo la curva $=0.751$.

gl: grados de libertad; Sig: significancia; OR: Odds ratio; IC: intervalo de confianza.

consumo de alcohol con respecto a los que no consumieron, mientras que las lesiones graves se concentraron (19.9\%) en los pacientes que reportaron consumo de alcohol más que en los sujetos que no lo consumieron $(7.8 \%)$.

\section{Variables predictoras del consumo de alcohol antes de la lesión}

La probabilidad de presentar lesiones con consumo previo de alcohol se incrementó cuatro veces más en los hombres que en las mujeres $(\mathrm{OR}=4.19$; IC95\% 2.52-6.99). Con respecto al horario de ocurrencia de la lesión, la probabilidad fue tres veces mayor en el horario nocturno que en el diurno $(\mathrm{OR}=3.17$; IC95\% 2.08-4.88). De las variables que se asociaron, la que tiene mayor probabilidad para explicar la variable consumo de alcohol previo a la lesión fue sexo, seguido por horario, día y escolaridad (cuadro 2).

\section{Variables predictoras de la gravedad de la lesión}

Los pacientes que consumieron alcohol tuvieron una probabilidad dos veces mayor (OR = 2.42; IC95\% 1.36-4.31) de presentar una lesión grave con respecto a los que no consumieron, y los hombres presentaron una probabilidad dos veces mayor de sufrir una lesión grave que las mujeres $(\mathrm{OR}=2.33$; IC95\% 1.12-4.84) (cuadro 3).

\section{Variables predictoras de las lesiones por violencia}

Con respecto a presentar una lesión relacionada con la violencia, de acuerdo con el número de copas consumidas, la mayor probabilidad (OR $=4.40$; IC95\% 2.17-8.90) se presentó en el consumo de seis a 15 copas, y disminuyó en el
Cuadro 3. Variables predictoras de la gravedad de la lesión, según análisis por regresión logística

\begin{tabular}{lccccc}
\hline Variables & $\chi^{2}$ Wald & $\mathrm{gl}$ & Sig & OR & $\mathrm{IC} 95 \%$ \\
\hline $\begin{array}{l}\text { Consumo de } \\
\text { Sí }\end{array}$ & 8.94 & 1 & 0.003 & 2.42 & $1.36-4.31$ \\
$\begin{array}{l}\text { Sí } \\
\text { No }\end{array}$ & & & & 1 & \\
$\begin{array}{l}\text { Sexo } \\
\text { Hombre }\end{array}$ & 5.13 & 1 & 0.023 & 2.33 & $1.12-4.84$ \\
Mujer & & & & 1 & \\
\hline
\end{tabular}

Análisis de regresión logística: Razón de verosimilitud $=336.407 \chi^{2}(2)=19.59$ $p=0.001$. Hosmer y Lemeshow $\chi^{2}(2)=0.206 p=0.902$. Porcentaje global de varianza explicada $=88.7 \%$. Área bajo la curva $=0.663$.

gl: grados de libertad; Sig: significancia; OR: Odds ratio; IC: intervalo de confianza.

consumo de 16 copas o más (OR = 3.00; IC95\% 1.56-5.80). Asimismo, en el horario nocturno se registró una probabilidad tres veces mayor (OR = 3.56; IC95\% 2.20-5.77) que en el diurno y el grupo de edad de 15 a 29 años presentó una probabilidad ligeramente mayor que en las personas con 30 años y más (OR = 1.68; IC95\% 1.05-2.70) (cuadro 4).

\section{DISCUSIÓN Y CONCLUSIÓN}

De acuerdo con el objetivo del estudio, los resultados confirman la asociación entre el consumo de alcohol y las lesiones. El porcentaje de pacientes lesionados que reportaron consumo de alcohol es similar al mostrado en otros estudios que utilizaron la misma metodología del Estudio Colaborativo sobre Alcohol y Lesiones de la OMS, como los realizados en Corea del Sur ${ }^{17}$ y Argentina, ${ }^{14}$ y mayor al reportado en un estudio multicéntrico realizado en países latinoamericanos que abarcó a Guatemala, Guyana, Nicaragua, Panamá y la República Dominicana. ${ }^{16}$ Aun cuando son países que observan un nivel de desarrollo similar, dicha diferencia se po-

Cuadro 4. Variables predictoras de las lesiones por violencia, según análisis por regresión logística

\begin{tabular}{|c|c|c|c|c|c|}
\hline Variables & $\chi^{2}$ Wald & gl & Sig. & OR & IC95\% \\
\hline \multicolumn{6}{|c|}{ Número de copas } \\
\hline 1 a 2 & 4.16 & 4 & 0.041 & 3.47 & $1.05-11.45$ \\
\hline 3 a 5 & 6.20 & 1 & 0.013 & 3.01 & $1.26-7.17$ \\
\hline 6 a 15 & 16.97 & 1 & 0.001 & 4.40 & $2.17-8.90$ \\
\hline 16 o más & 10.74 & 1 & 0.001 & 3.00 & $1.56-5.80$ \\
\hline \multicolumn{4}{|c|}{ Abstemios/no consumo previo } & 1 & \\
\hline \multicolumn{6}{|c|}{ Horario } \\
\hline Nocturno & 26.60 & 1 & 0.001 & 3.56 & $2.20-5.77$ \\
\hline Diurno & & & & 1 & \\
\hline \multicolumn{6}{|l|}{ Edad } \\
\hline 15 a 29 años & 4.65 & 1 & 0.031 & 1.68 & $1.05-2.70$ \\
\hline$\geq 30$ años & & & & 1 & \\
\hline
\end{tabular}

Análisis de regresión logística: Razón de verosimilitud $=448.716 \chi^{2}(6)=85.17$ $p=0.001$. Hosmer y Lemeshow $\chi^{2}(5)=1.96 p=0.854$. Porcentaje global de varianza explicada $=80.4 \%$. Área bajo la curva $=0.768$.

gl: grados de libertad; Sig: significancia; OR: Odds ratio; IC: intervalo de confianza. 
dría explicar a partir de los diferentes hábitos de consumo, de una mayor permisividad social y del incumplimiento de las políticas públicas y de la normatividad relacionadas con el alcohol.

Asimismo, en los estudios realizados en Pachuca, capital del Estado de Hidalgo ${ }^{11}$ y en la Ciudad de México, ${ }^{12}$ los porcentajes de consumo de alcohol fueron menores al obtenido en esta investigación, lo que podría deberse a las posibles diferencias en la observancia de la reglamentación sobre el consumo de alcohol en los diferentes Estados del país y al incremento general en el consumo de alcohol, de acuerdo con lo reportado en los últimos años en las encuestas nacionales, ya que los estudios en Pachuca y en la Ciudad de México fueron efectuados en 1996 y 2002, respectivamente.

Referente a la distribución por sexo y grupo de edad de los lesionados, los resultados obtenidos mostraron consistencia con otros estudios similares realizados en los servicios de urgencias, ${ }^{14,16,19,22}$ en los cuales la mayoría de los pacientes con lesiones fueron hombres y jóvenes de entre 15 y 29 años. Se trata de una situación que debe prevenirse enfatizando en las lesiones derivadas del consumo de alcohol, tomando en cuenta las consecuencias negativas de estos sucesos entre los que destacan la pérdida de años de vida saludable por discapacidad, los gastos catastróficos para sí o para terceros.

En concordancia con lo anterior, los hombres mostraron una probabilidad tres veces mayor de presentar una lesión tras el consumo de alcohol que las mujeres. Estos resultados coinciden con la mayoría de los estudios realizados en los servicios de urgencias que han señalado que el pertenecer al sexo masculino aumenta la probabilidad de sufrir un traumatismo relacionado con el alcohol, 9,10,13,14,19 lo que refuerza sus conclusiones sobre los roles de género y sus implicaciones en la salud, debido a que los hombres tienden a consumir alcohol con mayor frecuencia y en mayor cantidad que las mujeres, como señala el análisis de Treviño. ${ }^{23}$ Esta conducta se vincula a una expresión de masculinidad mediante la cual muestran su resistencia, control y disposición a tomar riesgos, aumentando la probabilidad de consecuencias adversas como las lesiones. También, ciertos patrones de consumo, como el episódico, suelen ser socialmente más aceptados para los hombres que para las mujeres., ${ }^{9,10,23} \mathrm{Sin}$ embargo, debe tenerse en cuenta que el nivel y patrón de uso de alcohol entre mujeres jóvenes tiende a ser cada vez más semejante al de los hombres de su misma edad.

Con respecto a la escolaridad, en el estudio se encontró una mayor probabilidad de consumo de alcohol en los pacientes con estudios de licenciatura o más; estos resultados difieren de otros que reportaron una mayor probabilidad de consumo en los niveles educativos más bajos ${ }^{9,24}$ y coincide con los resultados de un estudio realizado en Brasil que mostró una asociación entre el abuso de alcohol y las variables de escolaridad y clase social, vinculando la educación superior con aquel. ${ }^{25}$
Como era de esperarse, el horario nocturno y los fines de semana fueron los más proclives a que se presentaran las lesiones relacionadas con el consumo de alcohol y permite concluir que en estos períodos las personas tienen más tiempo libre, enfrentan menos responsabilidades y existe un mayor espacio de permisividad social, por lo que factores de índole cultural relacionados con contextos recreativos facilitan el consumo de alcohol episódico intenso, lo que conduce a una mayor práctica de comportamientos de riesgo.

$\mathrm{El}$ alcohol es considerado como un factor causal en las lesiones, ya sea porque propicia un comportamiento agresivo que deriva en el ejercicio de la violencia o porque reduce la coordinación motriz y la capacidad de respuesta que puede ocasionar accidentes. Aunque más de la mitad de los lesionados de este estudio, que reportaron consumo de alcohol presentaron lesiones no intencionales provocadas por accidentes, no se puede soslayar la importancia de la frecuencia de lesiones intencionales resultantes de la violencia, misma que también se ha encontrado en otros estudios realizados. ${ }^{26}$

Los resultados mostraron que la probabilidad de sufrir una lesión relacionada con la violencia fue mayor en el horario nocturno y en los jóvenes de 15 a 29 años de edad. Además de los factores de índole social y cultural antes mencionados, también se deben tomar en cuenta las razones de orden biológico, ya que se ha demostrado que el proceso de maduración del cerebro humano termina, en promedio, después de los 20 años de edad, y que el área frontal relacionada con la toma de decisiones es la última en madurar, de ahí que los jóvenes tengan una menor capacidad para distinguir la gravedad de los riesgos por lo que suelen presentar comportamientos temerarios que los exponen a ser agredidos o a agredir a otros, más aún si ingieren bebidas alcohólicas. $3,15,23,27$

Estudios realizados en los servicios de urgencias, ${ }^{15,17,19}$ reportaron que en cuanto al número de copas consumidas, la mayor probabilidad se mostró en el rango de seis a 15 copas; sin embargo, es conveniente precisar que aun en los niveles bajos - como el consumo de una a dos copas - la probabilidad de presentar una lesión relacionada con la violencia fue mayor que en aquellos que no consumieron. La mayoría de los estudios coinciden en señalar que el riesgo se mantiene relativamente estable durante un máximo de seis copas y que aumenta de manera brusca ante el incremento de las copas consumidas. ${ }^{16}$

Con respecto a la gravedad de la lesión, los resultados revelan que el consumo de alcohol en hombres aumenta la probabilidad de que ésta sea grave. Este hallazgo conduce a la reflexión sobre la necesidad de diseñar, instrumentar y reforzar acciones para reducir el consumo de alcohol, principalmente en hombres jóvenes, en fines de semana y en horario nocturno, con el fin de apoyar la prevención de lesiones, principalmente de aquellas que, por su gravedad, requieren de hospitalización, generan una mayor demanda de recursos para su atención y ponen en peligro la vida. 
Este es el primer trabajo realizado en Xalapa que aborda el problema del consumo de alcohol y la ocurrencia de lesiones en pacientes que acuden a los servicios de urgencias de dos hospitales públicos. En el marco de la Estrategia Mundial para reducir el uso nocivo de alcohol y ante los resultados de este trabajo, resulta ineludible la necesidad de revisar y fortalecer las políticas públicas destinadas a la prevención, atención y tratamiento del abuso de alcohol. Se trata de impulsar políticas públicas que incidan en los patrones de consumo y en los servicios de salud disponibles para tratar a los bebedores con problemas. ${ }^{28}$

Adicionalmente, aun cuando en el Estado de Veracruz no existe un marco legal que atienda esta problemática, resalta la necesidad de involucrar al personal de salud que atiende a la población que llega a los servicios de urgencias como resultado de una lesión vinculada al consumo de alcohol, en el desarrollo de estrategias de detección, tales como el uso de pruebas de tamizaje para detectar o confirmar su consumo y la canalización de los casos positivos con el fin de que los expertos realicen intervenciones breves o tratamiento, según el caso, para reducir el consumo abusivo de alcohol y promover uno responsable para prevenir las consecuencias negativas, especialmente las relacionadas con la generación de lesiones. ${ }^{29,30}$ Los resultados de estas intervenciones podrían evaluarse en futuras investigaciones.

Finalmente, se sugiere profundizar en otros aspectos ligados a la relación entre el consumo de alcohol y la presencia de lesiones. Tal es el caso de la investigación de los fundamentos y aplicación de las políticas públicas y la normatividad, ${ }^{29}$ la permisividad social, así como la disponibilidad y la accesibilidad al alcohol como factores que favorecen su consumo. ${ }^{29}$

\section{Limitaciones}

Aunque se obtuvo una muestra representativa de los pacientes atendidos en urgencias en los dos hospitales incluidos en el estudio, una limitación es que dicha muestra no fue representativa de otras unidades médicas de esta misma ciudad o de otras regiones del país. Asimismo, los resultados no incluyen a los lesionados que fallecieron a causa de la lesión antes de llegar al hospital o aquellos que no solicitaron atención médica en el servicio de urgencias.

\section{Financiamiento}

Ninguno.

\section{Conflictos de interés}

Los autores declaran no tener ningún conflicto de intereses.

\section{Agradecimientos}

Al doctor Guilherme Borges por su asesoría en el desarrollo de esta investigación; a las autoridades de los hospitales participantes por permitir el acceso a sus instalaciones $\mathrm{y}$, en especial, al personal del servicio de Urgencias por su apoyo para la realización de este trabajo y a los pacientes y a sus familias, por su apertura y colaboración.

\section{REFERENCIAS}

1. World Health Organization. Global status report on alcohol and health. Ginebra: 2014.

2. Medina-Mora ME, Villatoro-Velázquez JA, Fleiz-Bautista C, Téllez-Rojo MM et al. Encuesta Nacional de Adicciones 2011: Reporte de alcohol. México DF: Instituto Nacional de Psiquiatría Ramón de la Fuente Muñiz; Instituto Nacional de Salud Pública; Secretaría de Salud; 2012.

3. Andreuccetti G, Gawryszewski V, Diehl A, Monteiro M et al. Papel causal del alcohol en la violencia interpersonal y los traumatismos por accidentes de tránsito en las Américas. En: Cherpitel CJ, Borges G, Giesbrecht N, Monteiro M et al. (eds.) Prevención de los traumatismos relacionados con el alcohol en las Américas: de los datos probatorios a la acción política. Washington, DC: OPS; 2013, pp.29-38.

4. Rehm J, Monteiro M. Alcohol consumption and burden of disease in the Americas, implications for alcohol policy. Pan American J Public Health 2005;18(4/5):241-248.

5. Monteiro MG, Telles-Dias $P$, Inglez-Dias A. Epidemiología del uso del alcohol y otras sustancias psicoactivas en América Latina y el Caribe. En: Monteiro MG, Telles-Dias P, Inglez-Dias A (eds.). Epidemiología de los trastornos mentales en América Latina y el Caribe. Washington, DC: Organización Panamericana de la Salud; 2009; pp.177-193.

6. Rehm J, Baliunas D, Borges G, Graham K et al. The relation between different dimensions of alcohol consumption and burden of disease: an overview. Addiction 2010;105:817-843.

7. Gutiérrez JP, Rivera-Dommarco J, Shamah-Levy T, Villalpando-Hernández $S$ et al. Encuesta Nacional de Salud y Nutrición 2012. Resultados nacionales. Cuernavaca, México: Instituto Nacional de Salud Pública (MX); 2012.

8. Schmidt LA, Mäkelä P, Rehm J, Room R. Alcohol: equity and social determinants. En: Blas E, Kurup AS (eds.). Equity, social determinants and public health programmes. Ginebra: WHO; 2010; pp.11-25.

9. Korcha R, Cremonte M. Comparación de las características demográficas como factores predictivos del consumo de alcohol y de traumatismos por nivel de ingresos del país. En: Cherpitel CJ, Borges G, Giesbrecht $\mathrm{N}$, Monteiro $\mathrm{M}$ et al. (eds.). Prevención de los traumatismos relacionados con el alcohol en las Américas: de los datos probatorios a la acción política. Washington, DC: OPS; 2013; pp.109-118.

10. Wilsnack RW, Vogeltanz ND, Wilsnack SC, Harris TR et al. Gender differences in alcohol consumption and adverse drinking consequences: cross cultural patterns. Addiction 2000;95(2):251-265.

11. Borges G, Medina-Mora ME, Cherpitel C, Casanova L et al. Consumo de bebidas alcohólicas en pacientes de los servicios de urgencias de la ciudad de Pachuca, Hidalgo. Salud Pública Mex 1999;41:3-11.

12. Borges G, Mondragón L, Casanova L, Rojas E et al. Substance and alcohol use and dependence in a sample of patients from an emergency department in Mexico City. Salud Mental 2003;26(1):23-31.

13. Macdonald S, Cherpitel C, Borges G, De Souza A et al. The criteria for causation of alcohol in violent injuries based on emergency room data from six countries. Addictive Behaviors 2005;30:103-113.

14. Borges G, Cherpitel C, Orozco R, Bond J et al. Acute alcohol use and the risk of non-fatal injury in sixteen countries. Addiction 2006;101:993-1002.

15. Borges G, Orozco R, Cremonte $\mathbf{M}$, Buzi-Figlie $\mathbf{N}$ et al. Alcohol and violence in the emergency department: a regional report from the WHO collaborative study on alcohol and injuries. Salud Publica Mex 2008;50(suppl 1):S6-S11.

16. Borges G, Orozco R, Monteiro M, Cherpitel CJ et al. Risk of injury after alcohol consumption from case-crossover studies in five countries from the Americas. Addiction 2012;108:97-103. 
17. Chou P, Chun S, Smith S, Ruan J et al. Episodic heavy drinking, problem drinking and injuries. Results of the WHO/NIAAA Collaborative Emergency Room Study in South Korea. Alcohol 2012;46(5):407413.

18. Andreuccetti G, Carvalho HB, Korcha R, Ye Y et al. A review of emergency room studies on alcohol and injuries conducted in Latin America and the Caribbean region. Drug Alcohol Review 2012;31:737-746.

19. Korcha RA, Cherpitel CJ, Witbrodt J, Borges G et al. Violence-related injury and gender: The role of alcohol and alcohol combined with illicit drugs. Drug Alcohol Review 2014;33:43-50.

20. Instituto Nacional de Salud Pública. Encuesta Nacional de Salud y Nutrición 2012. Resultados por entidad federativa, Veracruz. Cuernavaca, México: 2013.

21. Borges G, Cherpitel CJ, Orozco R, Bond J et al. Multicentre study of acute alcohol use and non-fatal injuries: data from the WHO collaborative study on alcohol and injuries. Bull World Health Org 2006;84:453-460.

22. Borges G, Cherpitel CJ. Estudios sobre servicios de urgencias realizados en las Américas: métodos y contexto. En: Cherpitel CJ, Borges G, Giesbrecht N, Monteiro M et al. (eds.). Prevención de los traumatismos relacionados con el alcohol en las Américas: de los datos probatorios a la acción política. Washington, DC: OPS; 2013; pp.53-56.

23. Treviño-Siller S, Villanueva-Borbolla M, Marcelino-Sandoval Y, Álvarez-Guillén F. Masculinidad, accidentes viales y políticas públicas. En: Figueroa JG (coord.). Políticas públicas y la experiencia de ser hombre. México, DF: El Colegio de México; 2014; pp.209-245.

24. Bloomfield K, Grittner U, Kramer S, Gmel G. Social inequalities in alcohol consumption and alcohol-related problems in the study countries of the EU concerted action "Gender, culture and alcohol problems: a multi-national study". Alcohol Alcoholism 2006;41(Suppl):i26-i36.

25. Almeida-Filho N, Lessa I, Magalhães L, Araújo MJ et al. Social inequality and alcohol consumption-abuse in Bahia, Brazil. Interactions of gender, ethnicity and social class. Soc Psychiatry Psychiatr Epidemiol 2005;40:214-222.

26. Ye Y, Cherpitel CJ, Macdonald S. Traumatismos relacionados con el consumo de alcohol en las Américas: variaciones en función de la causa y el país. En: Cherpitel CJ, Borges G, Giesbrecht N, Monteiro M et al. (eds.). Prevención de los traumatismos relacionados con el alcohol en las Américas: de los datos probatorios a la acción política. Washington, DC: OPS; 2013; pp.65-74.

27. Híjar-Medina M, Flores-Regata L, Valdez-Santiago R, Blanco J. Atención médica de lesiones intencionales provocadas por la violencia familiar. Salud Publica Mex 2003;45:252-258.

28. Babor T, Caetano R, Casswell S, Edwards G et al. Alcohol, no ordinary commodity: Research and public policy. Oxford: Oxford University Press; 2003; p.7.

29. Del Bosque J, Fernández C, Fuentes A, Díaz D et al. Hacia una mejor respuesta ante el problema del abuso de bebidas con alcohol: el papel del sector salud. Salud Ment 2012;35:165-174.

30. Cremonte M, Monterio M, Cherpitel CJ. Intervenciones encaminadas a disminuir los traumatismos relacionados con el alcohol en el servicio de urgencias: el tamizaje, la intervención breve y el seguimiento. En: Cherpitel CJ, Borges G, Giesbrecht N, Monteiro M et al. (eds.). Prevención de los traumatismos relacionados con el alcohol en las Américas: de los datos probatorios a la acción política. Washington, DC: OPS; 2013; pp.177-187. 